\title{
Aplicación de urbanismo táctico en la Universidad de lbagué (Colombia)
}

\section{Implementing tactical urbanism at Universidad de lbagué (Colombia)}

\author{
Juanita Ciro Torres ${ }^{1}$ \\ Juan Guillermo Zuluaga Villermo² \\ Eduardo Peñaloza Kairuz ${ }^{3}$ \\ Carlos Augusto Kaffure Ruiz ${ }^{4}$
}

○

Recepción: 31/08/2020
(.)

Aprobación: 14/10/2020
(-)

Publicación: 18/12/2020

\section{Para citar este artículo:}

Ciro Torres, J., Zuluaga Villermo, J. G., Peñaloza Kairuz, E., \& Kaffure Ruiz, C. A. (2020). Aplicación de urbanismo táctico en la Universidad de Ibagué. Indagare, (8), 100-125. https://doi.org/10.35707/indagare/809

\section{(c) $(9)$}

\footnotetext{
${ }^{1}$ Grupo de investigación Semillero EnMiBus, Universidad de Ibagué, Colombia. ORCID: 0000-0002-2019-9091. Correo electrónico: juanitacirot@gmail.com

${ }^{2}$ Grupo de investigación GMAE, Universidad de Ibagué, Colombia. ORCID: 0000-0002-2236-6279. Correo electrónico: juan.zuluaga@unibague.edu.co

${ }^{3}$ Grupo de investigación Rastro Urbano, Universidad de Ibagué, Colombia. ORCID: 0000-0002-3016-4095. Correo electrónico: eduardo.penaloza@unibague.edu.co

${ }^{4}$ Grupo de Investigación en Estudios de Arquitectura y Ciudad, Universidad del Tolima, Colombia. ORCID: 0000-00016094-2962. Correo electrónico: carkaffure@ut.edu.co
} 


\section{Resumen}

En el siglo Xx, el modelo urbanístico tenía una configuración de ciudad dispersa y difusa, aquella donde imperaban los medios de transporte motorizado sobre los medios de movilidad activa que, en consecuencia, dejaban la carencia en infraestructura peatonal y ciclista. Por esta razón, se deben realizar modificaciones viales en beneficio de la seguridad y comodidad de los ciudadanos, con la creación de nuevos espacios urbanos para su desplazamiento. En desarrollo a esto, las personas han encontrado el medio adecuado para proponer soluciones a través de acciones temporales, económicas y creativas, que se identifican como urbanismo táctico.

Con el fin de implementar políticas de transporte sostenible, se elabora un diseño de urbanismo táctico en la calle 66 del barrio Ambalá en la ciudad de Ibagué, Colombia, contiguo a la Universidad de Ibagué. Este proceso se lleva a cabo, dado que la calle presenta serios problemas de movilidad que intervienen en la calidad de vida de las personas que habitan el sector y quienes hacen uso frecuente de la misma. El objetivo es analizar los beneficios e impactos sociales que se pueden generar luego de esta intervención, en aras de exponer la capacidad del urbanismo táctico para solucionar problemas de movilidad y espacio público.

\footnotetext{
Abstract

In the twentieth century, the urban model had a scattered and diffuse conformation, that one in which motorized transport prevailed over active mobility means and that, consequently, led to the lack of pedestrian and cycling infrastructure. For this reason, road modifications should be made to benefit safety and comfort of citizens, with the creation of new urban spaces for their movement. In response to this, people have found a suitable way to propose solutions through temporary, economic and creative actions, which are identified as tactical urbanism.

In order to implement sustainable transportation policies, a tactical urbanism design is drawn up on Calle 66, in Ambalá neighborhood, in the city of Ibagué, Colombia, nearby Universidad de Ibagué. This process is carried out, given that the street presents serious mobility problems that impact the quality of life of people who live in the area and use it frequently. The objective is focused on analyzing benefits and social impacts that can be generated after this intervention, for the sake of presenting the capacity of tactical urbanism to solve problems of mobility and public space.
} 


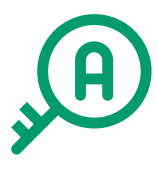

Palabras claves: Peatón, urbanismo, movilidad, urbanismo táctico, intervención, diseño.

Key words: Pedestrian, urbanism, mobility, tactical urbanism, intervention, design.

\section{Introducción}

El vertiginoso avance de la movilidad y el urbanismo han permitido el derecho inherente a la condición de ciudadano, por tanto, la ciudad se caracteriza por ser lugar de relación social, según el uso y tratamiento que se le dé al espacio público. En este sentido, es fundamental reflexionar sobre el diseño de las infraestructuras y el espacio público para mejorar la calidad de vida de los ciudadanos a través de la integración física.

En la sociedad actual, se deben crear diseños o estrategias que replanteen la concepción del espacio urbano, como un lugar de conectividad y movilidad universal donde se pueda lograr una integración urbana, social y cultural. A la vez, analizar cada uno de los factores relacionados con condiciones del entorno, seguridad, funcionalidad, comodidad y economía, con el fin de minimizar los conflictos potenciales generados por el aumento de vehículos, autobuses y motocicletas, que permitan una adecuada movilidad en función de seguridad vial y calidad de vida.

El proceso de crecimiento de la calle 66 en el barrio Ambalá ha estado ligado con el crecimiento exponencial del sector residencial de los barrios aledaños, la irregularidad por asentamientos sin regulación, lecturas acordes geográficas y la expansión en la presencia de estudiantes por parte de la Universidad de Ibagué -ícono regional en términos educativos-, donde se han presentado procesos de crecimiento tanto en el número de estudiantes como en el territorio del campus universitario. Lo anterior permite destacar el predominio de intereses particulares sobre lo colectivo en el territorio urbano.

Esta infraestructura es una de las calles locales que conecta en su gran mayoría con barrios residenciales y fue diseñada para tal fin. Sin embargo, ha generado ciertas limitaciones para su tránsito, que han sido detectadas debido a la demanda inducida por la Universidad de Ibagué. De esta manera, se ha demostrado que la calle no cuenta con la capacidad suficiente para responder ante estas solicitaciones, pero aun así se evidencia que solo se usa un carril para el tránsito de vehículos motorizados. Esta situación lleva a comprender que no existe movilidad porque la zona es inutilizada o dedicada netamente al parqueo en vía. A continuación, se presenta la geometría del tramo analizado (Figura 1). 
Figura 1. Geometría de la calle

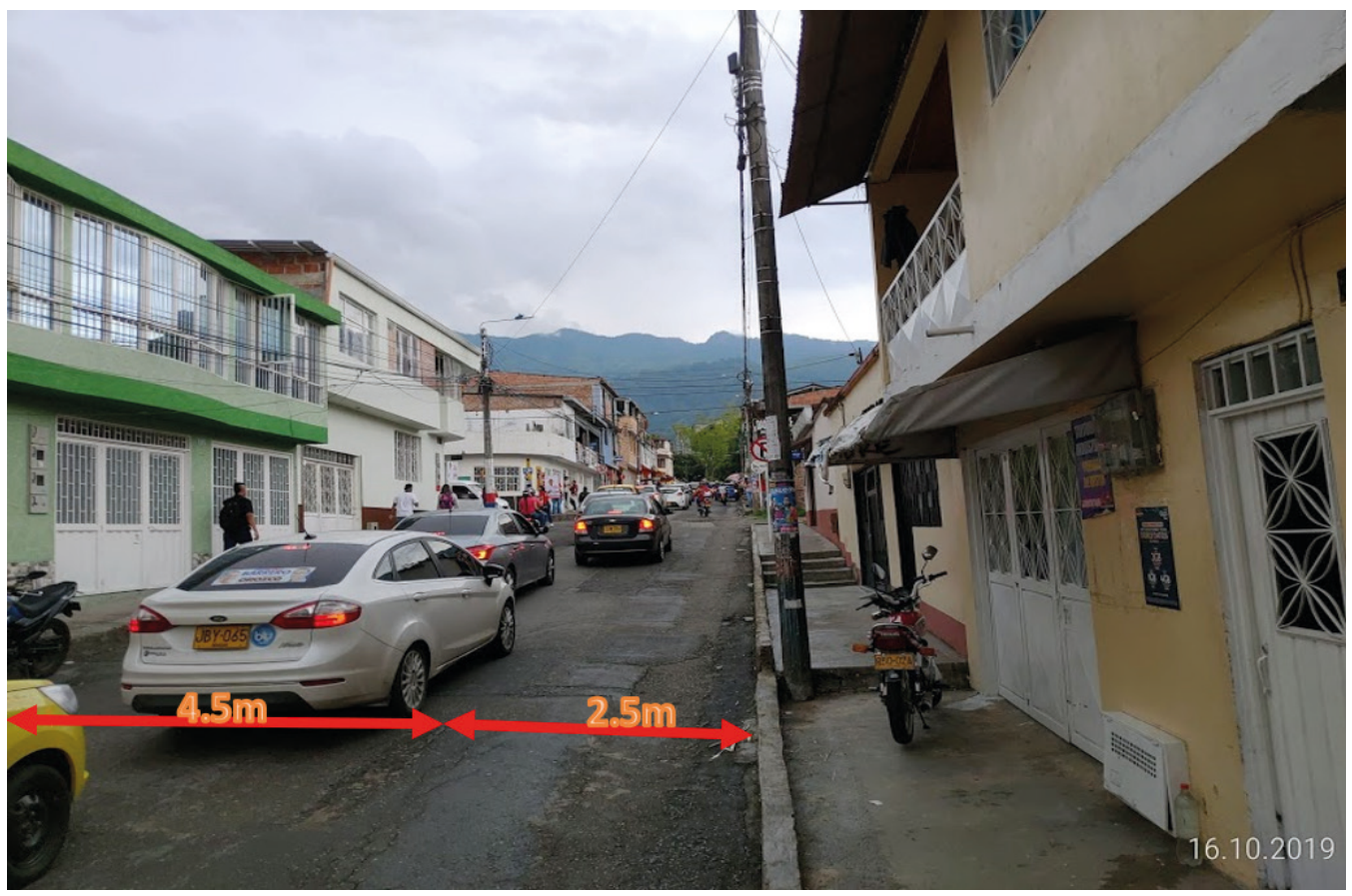

Fuente: autores (2020)

El tramo analizado tiene $150 \mathrm{~m}$ de largo y $7 \mathrm{~m}$ de ancho de calzada. De acuerdo con el análisis de uso de la calzada para que un camión pueda maniobrar se necesitan tan solo $4 \mathrm{~m}$, según la Guía global de diseño de calles (National Association of City Transportation Officials, 2016). Si se dejaran los $3 \mathrm{~m}$ sobrantes como zona dedicada a otros usos, se tendrán los valores que aparecen en la Figura 2. En la parte izquierda de ella se presentan los modos o infraestructura que cabría en el área, y en el lado derecho, las personas que moviliza o podrían usarla. 
Figura 2. Condiciones de utilización

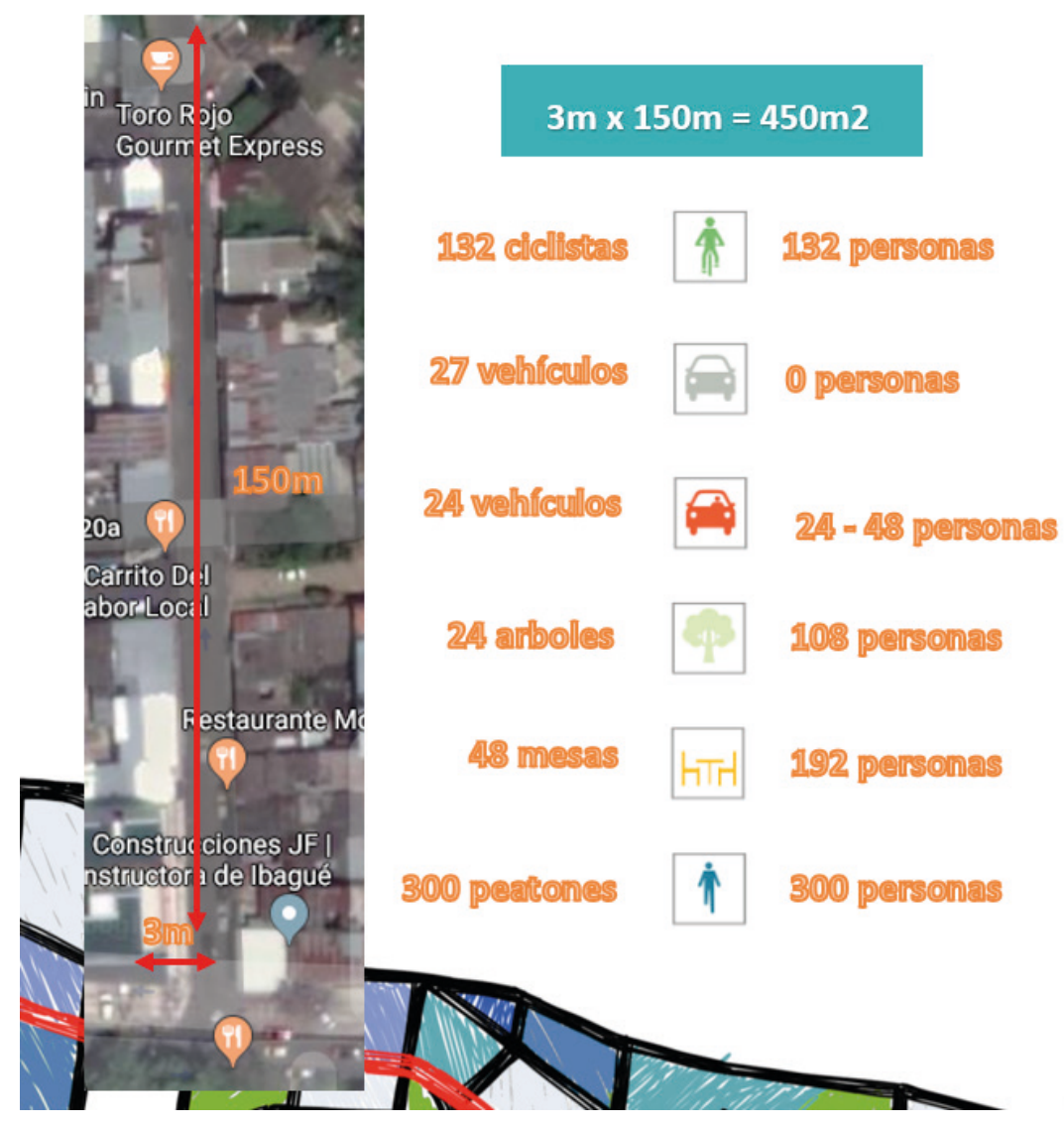

Fuente: autores (2020)

En este punto de la ciudad, en la calle 66, se ubica una de las principales entradas de la Universidad de Ibagué. Por sus dinámicas existe una gran variedad de locales comerciales atraídos por el alto flujo peatonal (estudiantes, funcionarios, universitarios y residentes de la localidad) y vehicular, que generan una demanda que responde ante actividades económicas, culturales y sociales en la zona. 
Figura 3. Distribución del espacio

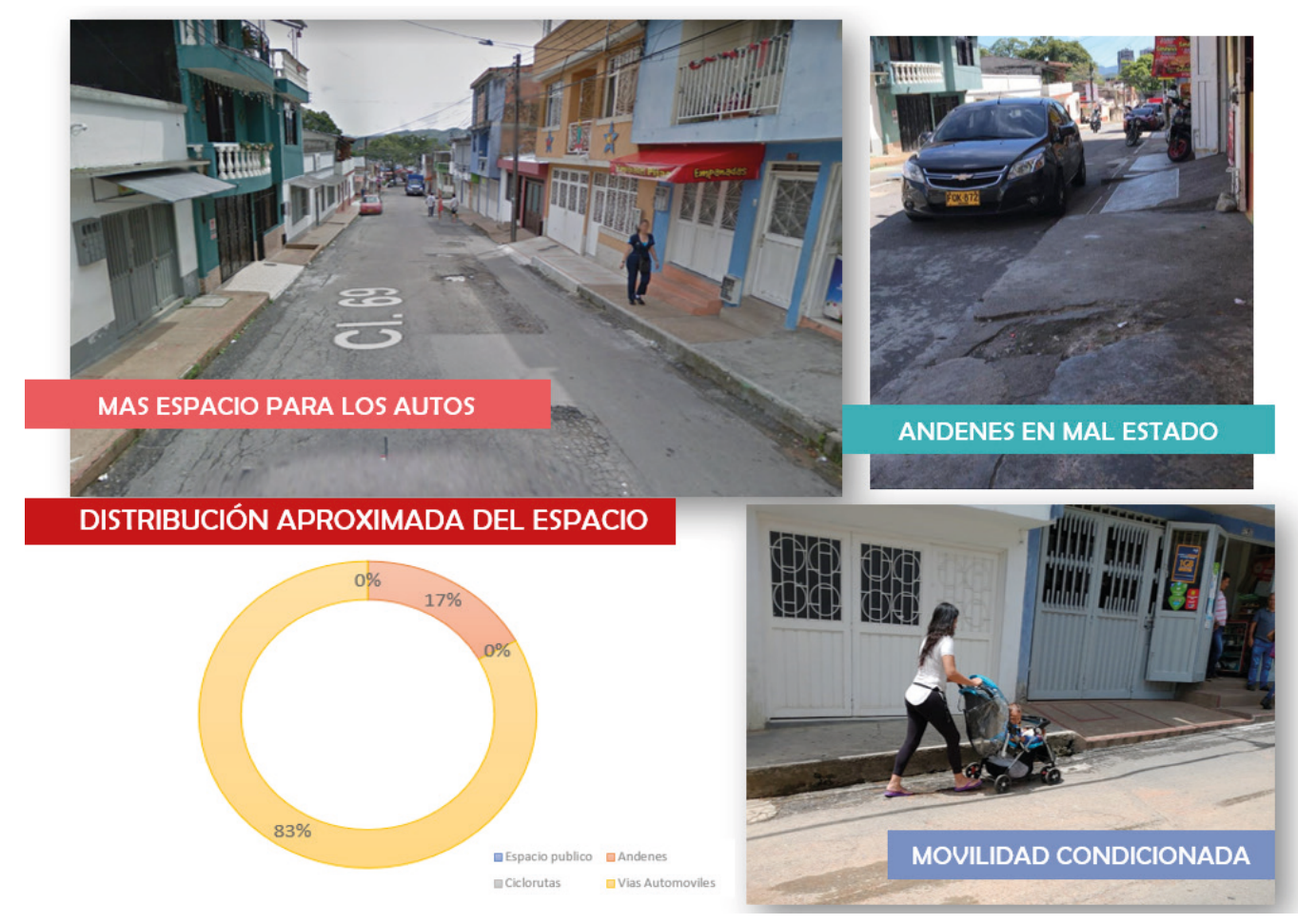

Fuente: autores (2020)

La distribución aproximada del espacio en la zona de estudio data que el 83 \% de la calle es designada para el tránsito de los automóviles y el $17 \%$ restante son andenes por donde transitan los peatones. Según observaciones, la movilidad de los peatones por los andenes se ve condicionada por su mal estado; los desniveles que presentan y las obstaculizaciones por el comercio, lo cual conlleva ser un espacio inutilizado que obliga a las personas a movilizarse por la carretera, que no solo es usada por el transporte motorizado, sino también de aparcamiento de los autos. Por consiguiente, es necesario implementar una política clara en el control del espacio público por parte de las entidades competentes.

En general, todas las personas somos usuarios activos de las vías, ya sea en condición de conductor, pasajero o peatón. Particularmente, uno de los desafíos que se presenta en el sector es el alto flujo de usuarios de la vía (vehículos, peatones, ciclistas, entre otros) que están en permanente conflicto, debido a la estrecha movilidad que se presenta en la calle y la poca señalización vial que genera riesgos en la seguridad e integridad de las personas que transitan por la zona. 


\section{Materiales y métodos}

Los experimentos en la calle pueden ser radicales, es decir, se pueden ejecutar prácticas que son diferentes a las dominantes, y en la medida en que implementan la proposición de que "las calles son para las personas, no para el tráfico" (Gehl, 2010, p. 93). Por consiguiente, este proyecto está planificado en fases, es un proceso continuo que permite articular la acción reflexiva y acción transformadora.

En la primera fase se identifica el problema a partir de la observación del gran volumen de peatones y el reducido espacio que contiene la calle para su tránsito, además de las altas velocidades con la que transitan los automóviles por la zona, poniendo en riesgo la seguridad e integridad de los usuarios más vulnerables de la vía. En la segunda fase se fundamenta la metodología cualitativa, que está basada en la observación y el estudio del comportamiento de las personas en el escenario social, con las condiciones viales actuales (Gehl \& Svarre, 2013). Se desarrolla el método descriptivo que permite identificar y delimitar el problema, la elaboración y construcción de los instrumentos, la observación y el registro de datos. Los formatos empleados se basan en las recomendaciones de la norma alemana de planeamiento de tráfico vehicular: Richtlinien für Lichtsignalanlagen (RILSA).

En la tercera fase se establecen los criterios de diseño basados en los resultados adquiridos por los instrumentos y los registros de datos, para caracterizar y establecer la demanda actual de la calle, determinando el volumen y tipo de vehículos que circulan. En la cuarta fase se define el diseño óptimo para la calle con base en los resultados de los estudios previos; en cada uno se prioriza el peatón, puesto que se crean espacios para ellos y para los biciusuarios, con el fin de promover la utilización de estos medios no motorizados para desplazarse. En la quinta fase se llevó a cabo la intervención de la calle con el diseño planteado, regido por las normas competentes.

Para finalizar, en la sexta fase se cumplió con un segundo acercamiento en campo, con el propósito de destacar cuantitativamente la importancia de la intervención, sus fortalezas, debilidades, oportunidades y amenazas en el diseño de la vía. Para la discusión y comparación de resultados, se presentan los pros y los contras del análisis de la intervención.

\subsection{Técnicas e instrumentos de recolección de datos}

Las técnicas de recolección de la información para este trabajo son aforos vehiculares, peatonales, cicloviales y de velocidades, que permitieron obtener los datos necesarios para el rediseño de la vía. 


\subsubsection{Volumen de transporte motorizado}

En el siguiente formato se registra el número de vehículos que pasan por la calle; están clasificados por sus características, que permiten establecer el tipo de vehículo: autos particulares, motos, taxis, buses escolares y camiones.

Tabla 1. Aforo Volumen motorizado

\begin{tabular}{|c|c|c|c|c|c|c|c|}
\hline \multirow{3}{*}{ Acceso } & \multirow{3}{*}{ Periodo } & \multirow{3}{*}{ Auto } & \multirow{3}{*}{ Taxi } & \multicolumn{4}{|c|}{ Otros } \\
\hline & & & & \multirow{2}{*}{ Moto } & \multicolumn{2}{|c|}{ Bus } & \multirow{2}{*}{ Camión } \\
\hline & & & & & Escolar & Otro & \\
\hline \multirow{2}{*}{\multicolumn{2}{|c|}{ De }} & $::::::::::$ & $::::::::::::::::::$ & $::::::::$ & $::::$ & $::::$ & $::::$ \\
\hline & & $:::::::::::$ & $::::::::::::::::::$ & $::::::::$ & $::::$ & $::::$ & $::::$ \\
\hline \multirow{2}{*}{\multicolumn{2}{|c|}{ A }} & $::::::::::$ & $::::::::::::::::::$ & $::::::::$ & $::::$ & $::::$ & $::::$ \\
\hline & & $::::::::::$ & $::::::::::::::::::$ & $::::::::$ & $::::$ & $::::$ & $::::$ \\
\hline
\end{tabular}

Subtotal

Fuente: Universidad de Ibagué

\subsubsection{Volumen no motorizado}

En el siguiente formato se registran los conteos de peatones y ciclistas caracterizados por género. A la vez, se subdividen y se destacan si son población infantil o adolescente, población adulta, adulto mayor o población con movilidad condicionada. Así, se analiza el sentido del flujo y el tipo de movimiento, de tal manera que se identifican los sitios críticos de flujos peatonales y ciclistas en el área de estudio.

Tabla 2. Aforo Volumen no motorizado

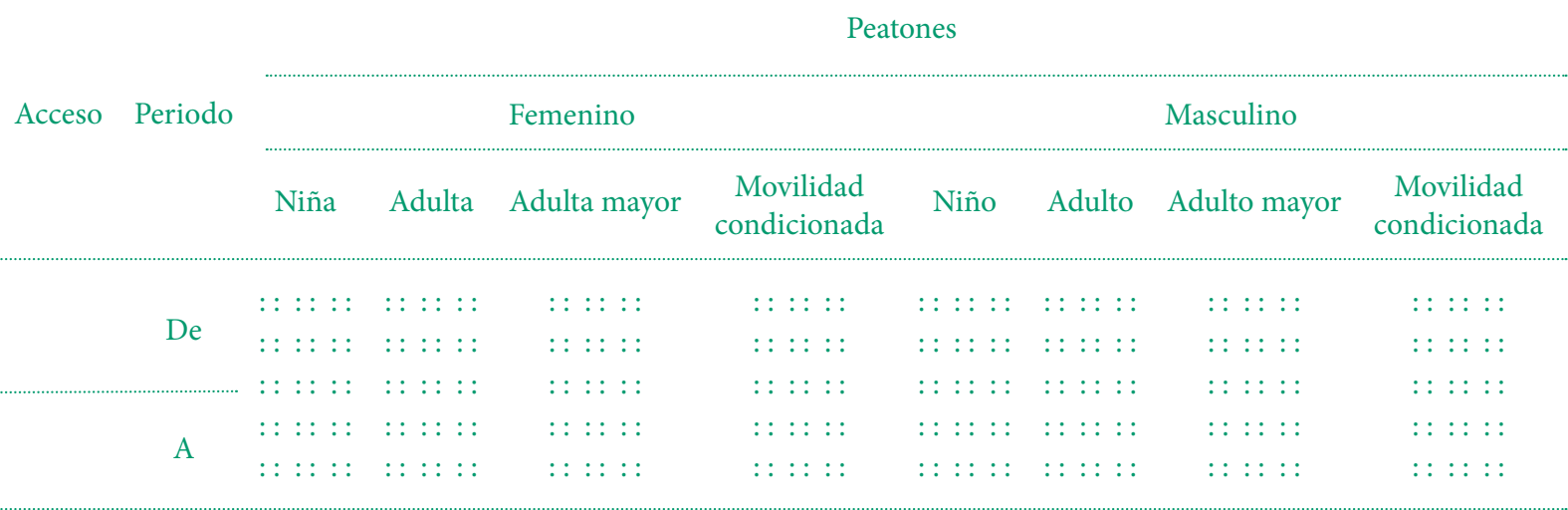




\section{Ciclistas}

\begin{tabular}{cccccc} 
Acceso Periodo & \multicolumn{3}{c}{ Memenino } \\
& Niña & Adulta & Adulta mayor & Niño & Adulto Adulto mayor \\
& & &
\end{tabular}

Subtotal

Fuente: Universidad de Ibagué

\subsubsection{Estudio de velocidades por vehículo}

Este aforo tiene por objetivo calcular la velocidad en marcha, que corresponde con la relación entre la distancia recorrida y el tiempo empleado de un vehículo para un viaje determinado, mientras está en movimiento sin contar demoras ni detenciones. Se mide la distancia entre un tramo de la vía y el tiempo recorrido de los transportes motorizados; en este caso, autos particulares, motos y taxis.

Tabla 3. Aforo Velocidad

Volumen: Velocidad

\begin{tabular}{|c|c|c|c|c|c|c|c|c|c|c|c|}
\hline \multicolumn{3}{|c|}{ Tipo de vehículo } & \multirow[t]{2}{*}{ Tiempo (s) } & \multicolumn{3}{|c|}{ Tipo de vehículo } & \multirow[t]{2}{*}{ Tiempo (s) } & \multicolumn{3}{|c|}{ Tipo de vehículo } & Tiempo (s) \\
\hline Carro & Moto & Taxi & & Carro & Moto & Taxi & & Carro & Moto & Taxi & \\
\hline
\end{tabular}




\subsubsection{Ocupación visual taxi}

En este formato se registra el número de pasajeros que transporta cada taxi. Esta información es importante por tratarse de la entrada a una universidad.

Tabla 4. Aforo Ocupación visual taxi

\begin{tabular}{|c|c|c|c|c|c|c|c|c|c|c|c|c|c|c|c|c|c|}
\hline \multirow{2}{*}{ \# } & \multicolumn{5}{|c|}{ Número de pasajeros } & \multicolumn{6}{|c|}{ Número de pasajeros } & \multirow{2}{*}{ \# } & \multicolumn{5}{|c|}{ Número de pasajeros } \\
\hline & 0 & 1 & 2 & 3 & $4+$ & & 0 & 1 & 2 & 3 & $4+$ & & 0 & 1 & 2 & 3 & $4+$ \\
\hline 1 & & & & & & 31 & & & & & & 61 & & & & & \\
\hline 2 & & & & & & 32 & & & & & & 62 & & & & & \\
\hline 3 & & & & & & 33 & & & & & & 63 & & & & & \\
\hline 4 & & & & & & 34 & & & & & & 64 & & & & & \\
\hline
\end{tabular}

Fuente: Universidad de Ibagué

\subsubsection{Ocupación visual automóvil}

En este formato se registra el número de personas que se transporta en un solo automóvil.

Tabla 5. Aforo Ocupación visual automóvil

\begin{tabular}{|c|c|c|c|c|c|c|c|c|c|c|c|c|c|c|c|c|c|}
\hline \multirow{2}{*}{ \# } & \multicolumn{5}{|c|}{ Número de pasajeros } & \multicolumn{6}{|c|}{ Número de pasajeros } & \multirow{2}{*}{ \# } & \multicolumn{5}{|c|}{ Número de pasajeros } \\
\hline & 0 & 1 & 2 & 3 & $4+$ & & 0 & 1 & 2 & 3 & $4+$ & & 0 & 1 & 2 & 3 & $4+$ \\
\hline 1 & & & & & & 31 & & & & & & 61 & & & & & \\
\hline 2 & & & & & & 32 & & & & & & 62 & & & & & \\
\hline 3 & & & & & & 33 & & & & & & 63 & & & & & \\
\hline 4 & & & & & & 34 & & & & & & 64 & & & & & \\
\hline
\end{tabular}

Fuente: Universidad de Ibagué

\section{Resultados}

\subsection{Aforos}

Con base en el esquema de la aproximación metodológica presentada, es turno de explicar los resultados de los acercamientos en campo con los aforos realizados antes y después de la intervención vial. 


\subsubsection{Antes de la intervención}

Se efectúan los conteos durante una semana, en horarios pico establecidos de 6:30 a 8:30, de 12:00 a 14:00 y de 17:00 a 19:00. Estos son horarios donde hay mayor flujo vehicular y peatonal.

- Volumen motorizado

Figura 4. Resultado primer periodo aforo motorizado

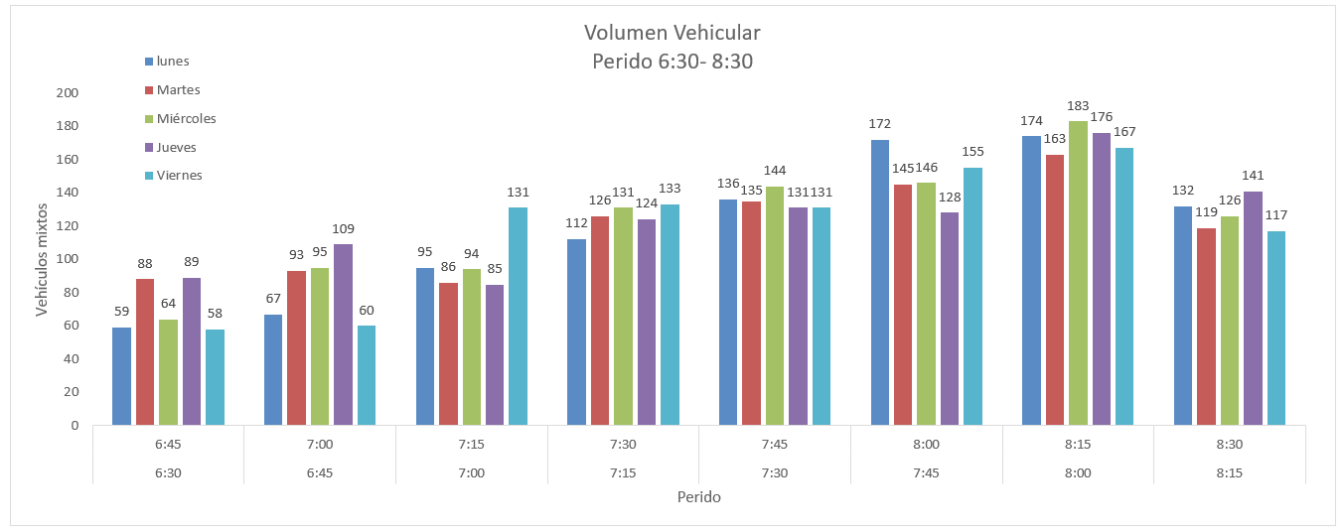

Fuente: autores

Figura 5. Resultado segundo periodo aforo motorizado

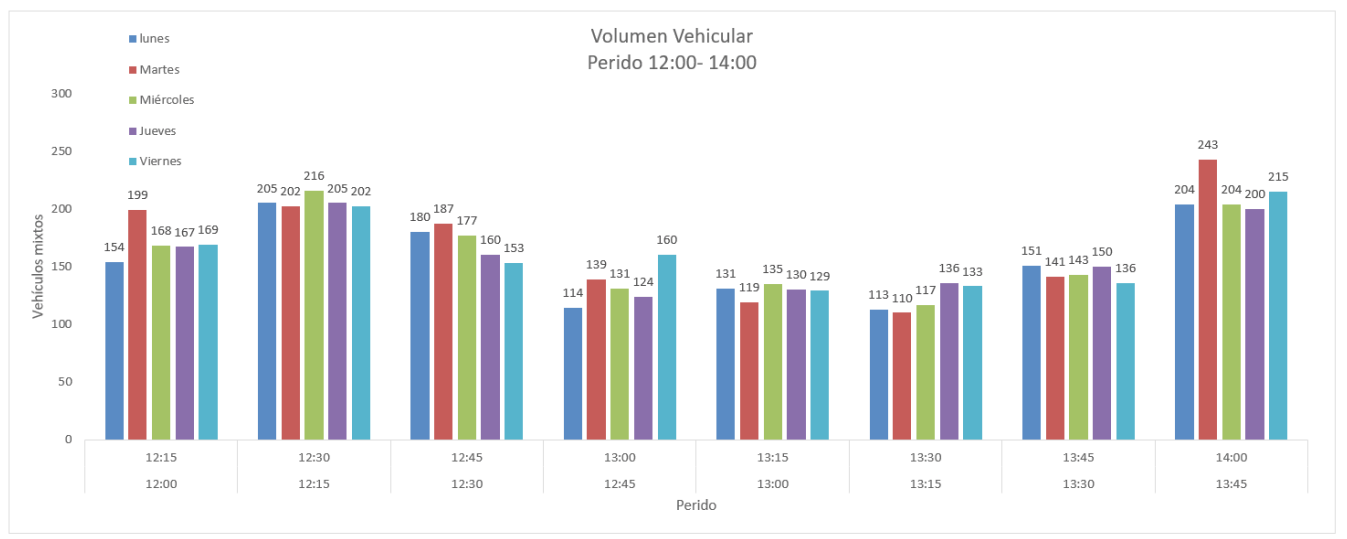

Fuente: autores 
Figura 6. Resultado tercer periodo aforo motorizado

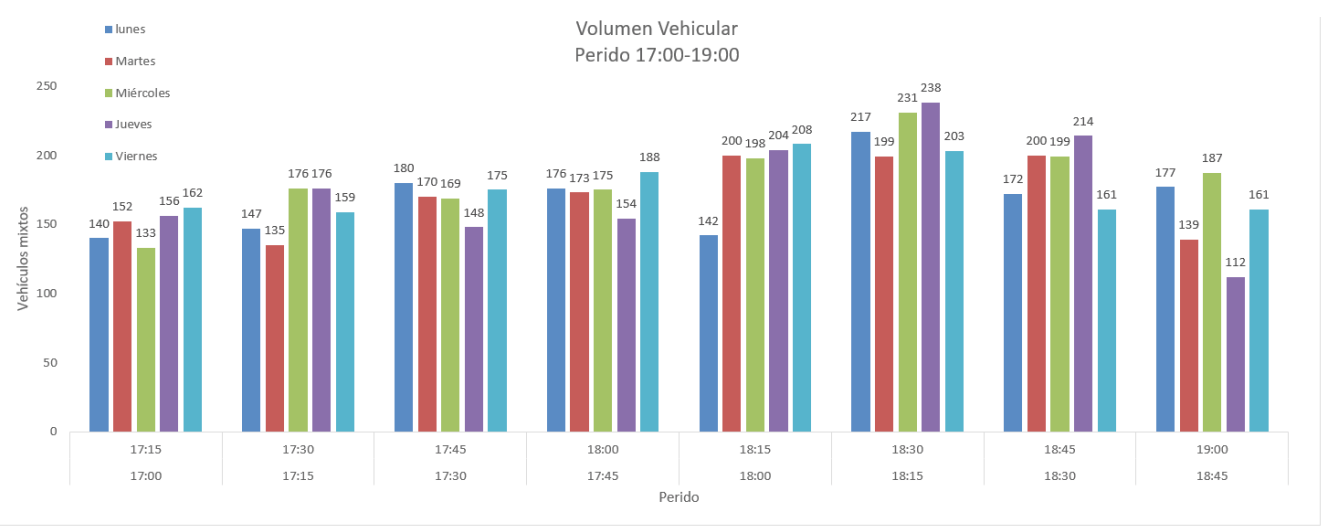

Fuente: autores

En el conteo de volumen motorizado se pudo apreciar lo siguiente: en la Figura 4, de 6:30 a 8:30, el flujo vehicular más alto se observa entre el horario de 8:00 a 8:15 del miércoles con una cantidad de 183 vehículos que transitan por la calle. Es claro que en este periodo es donde transitan más vehículos motorizados en el horario de la mañana en la semana. Esto se debe al ingreso de funcionarios y estudiantes.

El periodo con más flujo de vehículos motorizados es de 12:00 a 14:00, con un total de 243 para el martes (ver Figura 5). En ese mismo periodo se presenta un flujo de 163 peatones y biciusuarios (ver Figura 8). Este es un horario de conflicto porque muchos estudiantes y funcionarios salen de la universidad finalizando su primera jornada laboral; se desplazan hacia sus casas o contribuyen al comercio de la zona porque almuerzan en los sitios aledaños. El volumen vehicular de la noche se estudia en el periodo de 17:00 a 19:00 (ver Figura 6), en este horario se observa que el mayor flujo vehicular se presenta en el horario de 18:15 a 18:30 en toda la semana con un total de 238 vehículos el jueves, 231 el miércoles y 217 el lunes.

- Volumen no motorizado

Figura 7. Resultado primer periodo aforo no motorizado

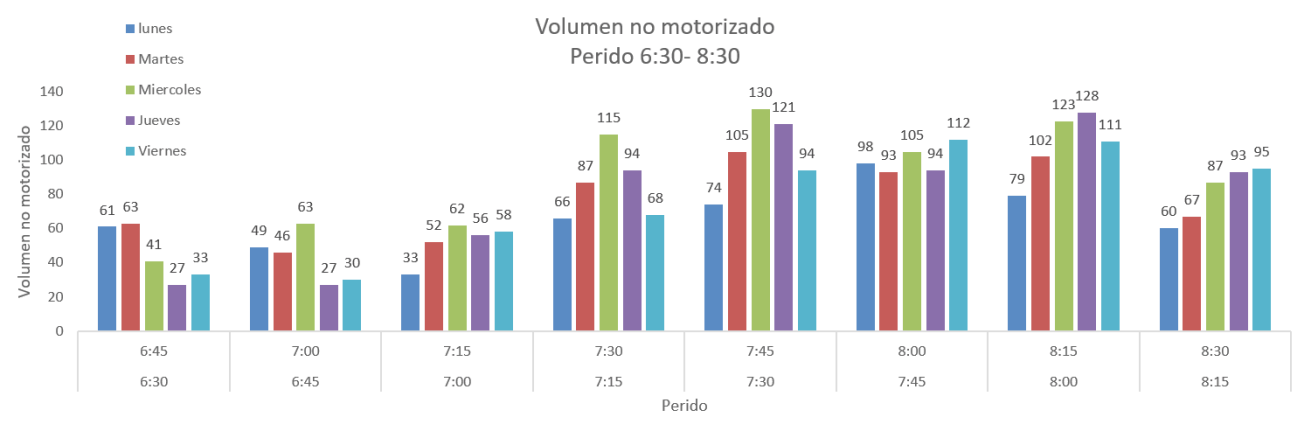

Fuente: autores 
Figura 8. Resultado segundo periodo aforo no motorizado

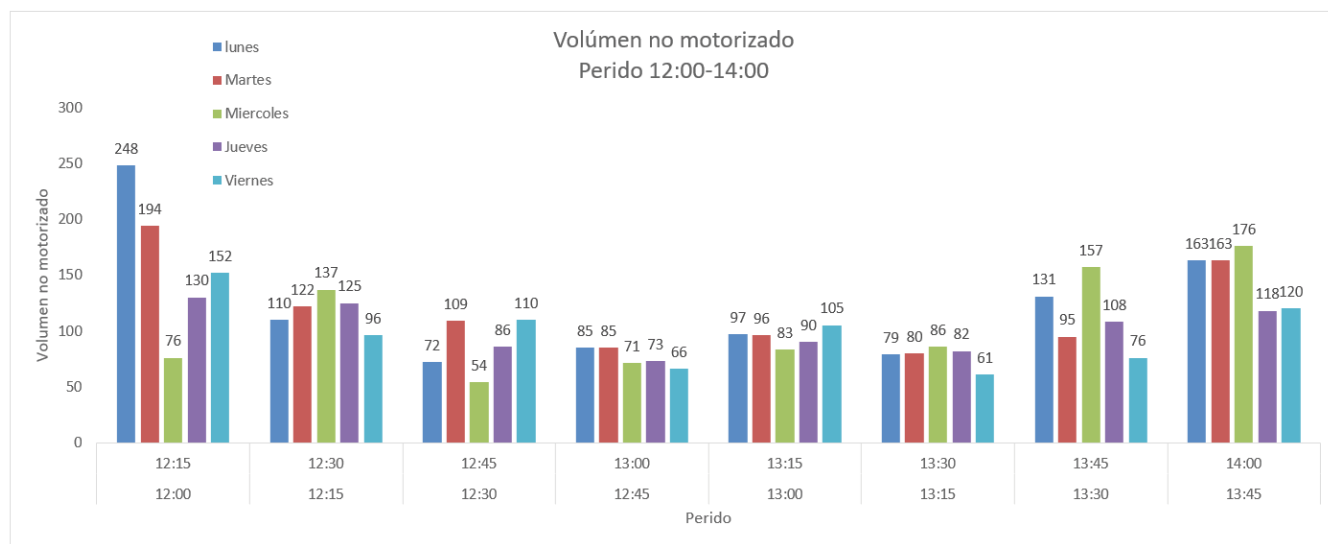

Fuente: autores

Figura 9. Resultado tercer periodo aforo no motorizado

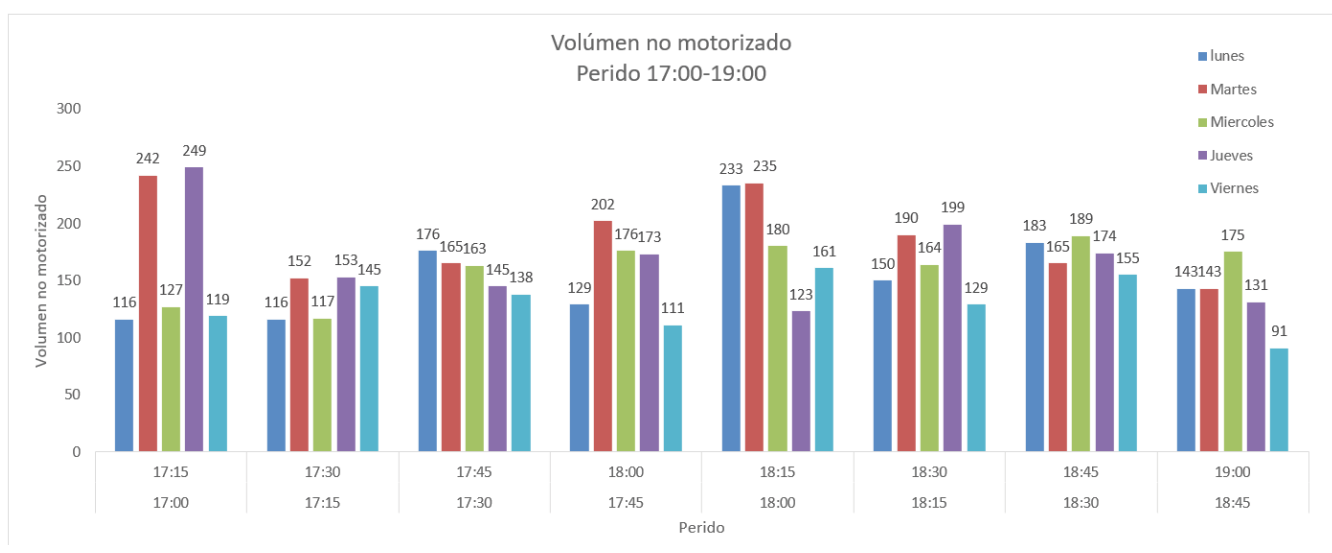

Fuente: autores

En este conteo se registraron los peatones y ciclistas que transitan por la calle. En la primera parte del día, en el periodo de 6:30 a 8:30, se presenta un total de 130 personas o biciusuarios para el miércoles en el horario de 7:30 a 7:45 (ver Figura 7). El día lunes, en el periodo de 12:00 a 14:00, se presenta un flujo no motorizado de 248 personas en el horario de 12:00 a 12:15; este el horario de ingreso para la segunda jornada laboral y académica del día (ver Figura 8).

El periodo con más flujo de no motorizados es 17:00 a 19:00, con un total de 249 personas o biciusuarios para el jueves (ver Figura 9). En ese mismo periodo se presenta un flujo de 156 motorizados (ver Figura 6), en él es evidente el conflicto que 
se presenta en esa franja de horario, entre motorizados y no motorizados; esto se debe a que a esa hora muchos estudiantes universitarios salen de clase y se dirigen a la calle 66 a comer o hacia sus casas. Adicional a esto, se desplazan muchos estudiantes escolares que salen de tomar cursos en el centro de idiomas de la universidad.

- Velocidad promedio

Figura 10. Resultado velocidad promedio automóvil

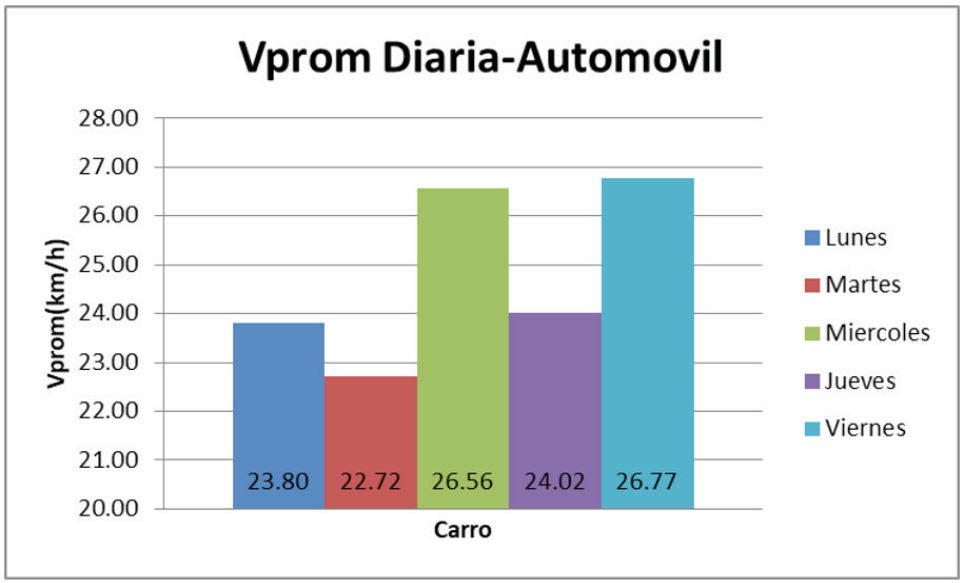

Fuente: autores

Figura 11. Resultado velocidad promedio moto

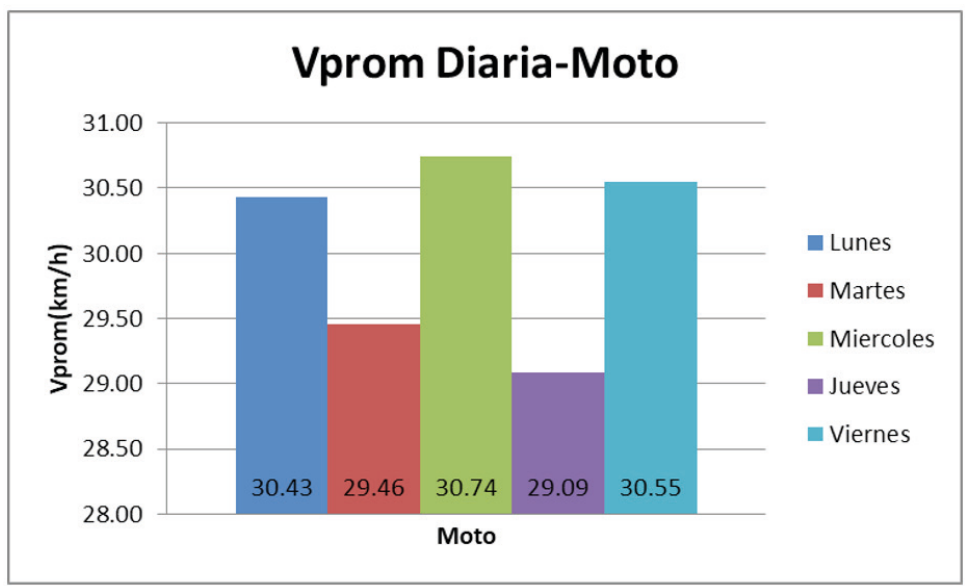

Fuente: autores 
Figura 12. Resultado velocidad promedio taxi

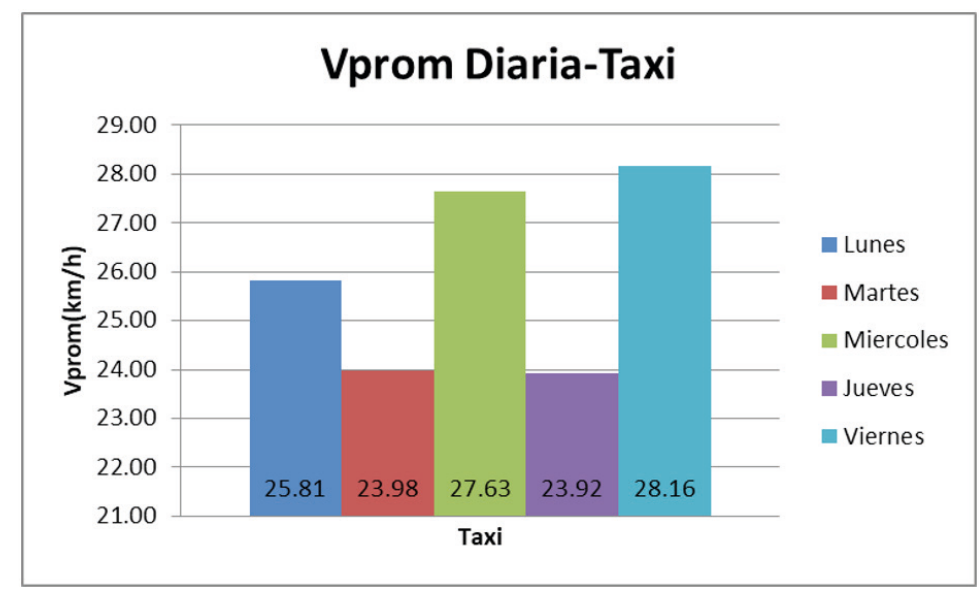

Fuente: autores

Según el artículo 106 del Decreto 0015 del 6 de enero de 2011 presentado por el Ministerio de Transporte colombiano, se establece que "la velocidad en zonas escolares y zonas residenciales será hasta de treinta (30) kilómetros por hora” (Decreto № 015, 2011, p. 1). Por eso, se afirma que tanto taxis como automóviles no exceden los límites de velocidad establecidos para la calle 66 (ver figuras 10 y 12). Por otra parte, las motocicletas exceden no por mucho la velocidad máxima, porque se presentan velocidades hasta de $30.74 \mathrm{~km} / \mathrm{h}$ (ver Figura 11). Se espera que con la implementación del diseño las velocidades reduzcan para salvaguardar la vida de los peatones y no motorizados que transitan por la calle.

- Conflicto vehículos-peatones sin intervención

Figura 13. Promedio vehicular y peatonal sin intervención

\begin{tabular}{|ccc|}
\hline $\begin{array}{c}\text { Pomedio } \\
\text { Peatones }\end{array}$ & $\begin{array}{c}\text { Promedio } \\
\text { Vehiculos }\end{array}$ & $p * v^{2}$ \\
\hline 719.5 & 800 & $4.6 . \mathrm{E}+08$ \\
\hline
\end{tabular}

Fuente: autores 
Figura 14. Resultado peatones-vehículos

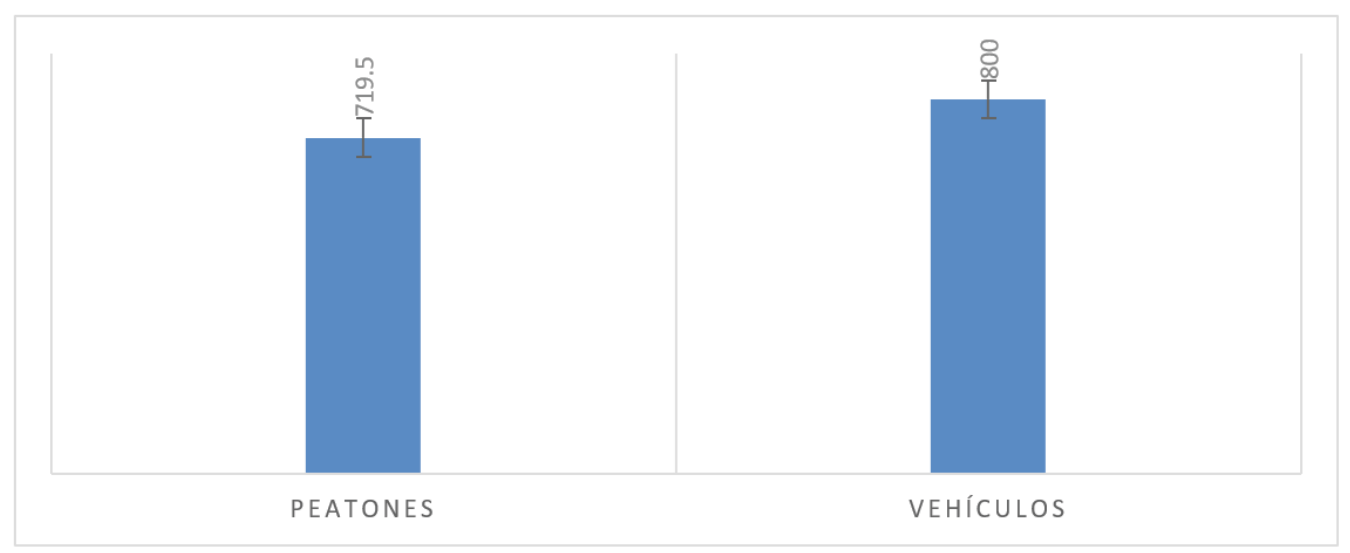

Fuente: autores

Con este resultado de $P V^{2}$, se logra medir el nivel de intensidad de conflicto vehículo-peatón, que se clasifica como un conflicto severo, porque el resultado es superior a $2^{\star} 10^{8}$. Esto significa que la cantidad de peatones es muy similar a la de los vehículos en la calle, por lo cual es necesaria la provisión de una zona de protección peatonal como lo indica la siguiente tabla:

Tabla 6. Determinación dispositivo según $P V^{2}$

\begin{tabular}{|c|c|c|c|}
\hline \multirow[t]{2}{*}{$P V^{2}$} & $\mathrm{P}$ & $\mathrm{V}$ & Recomendación \\
\hline & (peatones/hora) & (vehículos/hora) & preliminar \\
\hline \multirow{3}{*}{$\begin{array}{l}\text { Sobre } 10^{8} \text { (si no es posible } \\
\text { la provisión de una zona de } \\
\text { protección peatonal en la } \\
\text { calzada) }\end{array}$} & 50 a 1.100 & 300 a 500 & Paso cebra \\
\hline & 50 a 1.100 & sobre 500 & Semáforo peatonal con botonera \\
\hline & sobre 1.100 & sobre 300 & Semáforo peatonal con botonera \\
\hline \multirow{3}{*}{$\begin{array}{l}\text { Sobre } 2 \times 10^{8} \\
\text { (si existe o es necesaria la } \\
\text { provisión de una zona de } \\
\text { protección peatonal) }\end{array}$} & 50 a 1.00 & 400 a 750 & Paso cebra con isla o refugio peatonal \\
\hline & 50 a 1.100 & sobre 750 & Doble semáforo peatonal con refugio peatonal \\
\hline & sobre 1.100 & sobre 400 & Doble semáforo peatonal con refugio peatonal \\
\hline
\end{tabular}


El estudio de conflicto de peatones nos indica que es recomendable implementar una doble semaforización con refugio peatonal (ver Tabla 6). Debido a que es muy difícil implementar esta recomendación, se opta por diseñar pasos peatonales y una ciclobanda, como estrategias para disminuir el conflicto entre motorizados y no motorizados.

\subsection{Diseño urbanismo táctico}

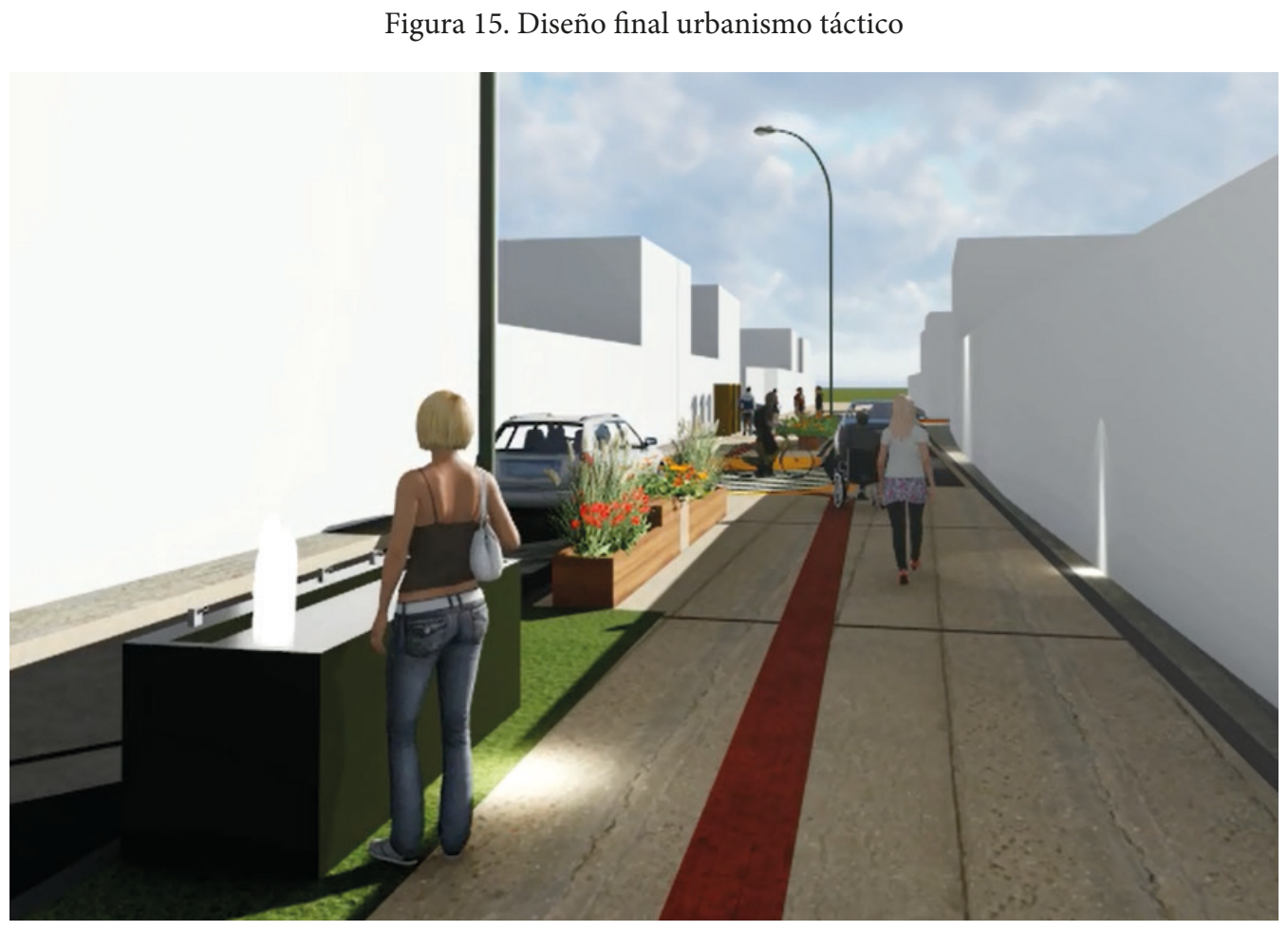

Fuente: autores

En el proyecto planteado se tuvieron en cuenta los principios de diseño según la Guía global de diseño de calles (National Association of City Transportation Officials, 2016), para así determinar y aplicar cada uno de los parámetros que son en realidad necesarios en una intervención óptima. Entre los elementos de diseño, se analiza la disposición de un paso a nivel, cebras, líneas de pare y un sendero peatonal. Las demarcaciones requeridas se realizan bajo los requerimientos establecidos en las Normas Técnicas Colombianas (NTC) y el Manual de señalización vial, adoptadas por el Ministerio de Transporte (2015). 
Figura 16. Diseño final urbanismo táctico
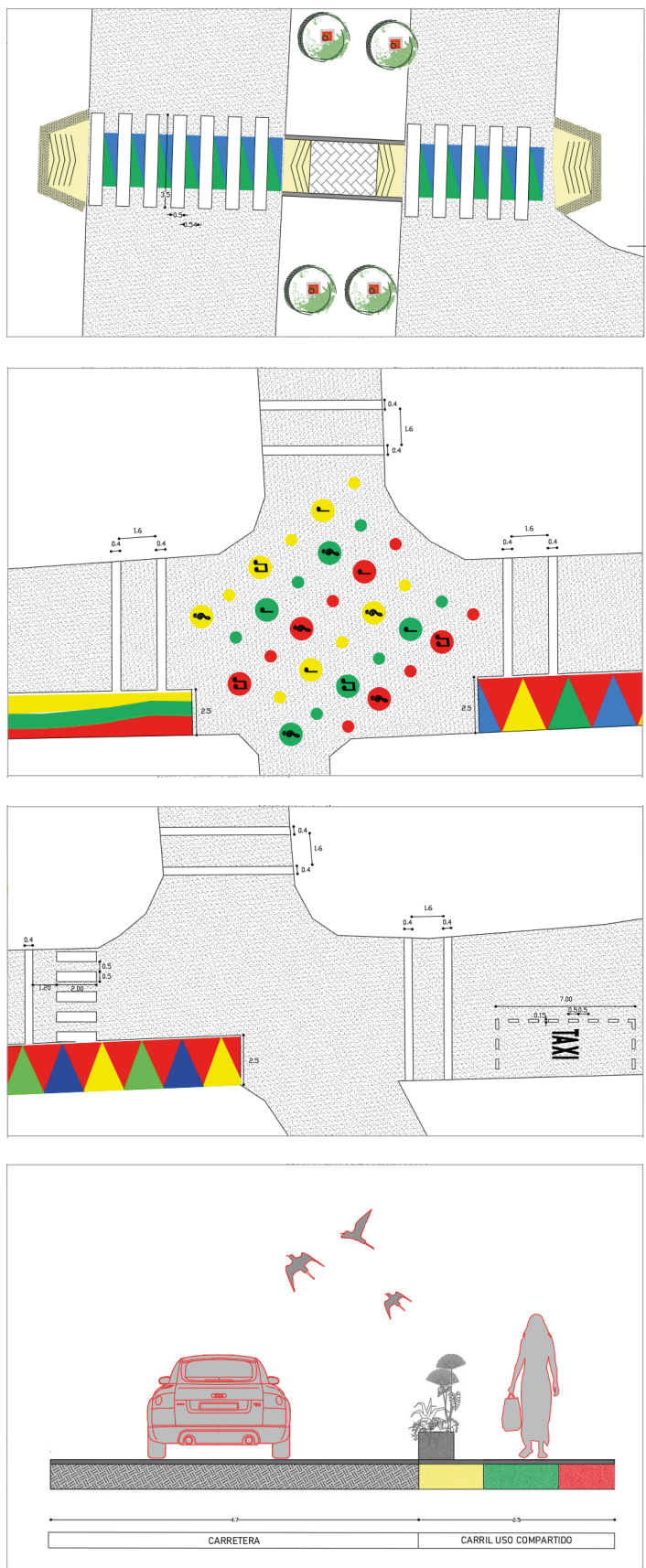

Fuente: autores 


\subsubsection{Aforos luego de la intervención}

Después de la intervención de urbanismo táctico realizado en la calle, se efectuó un segundo acercamiento en campo, para analizar la eficiencia de la intervención ejecutada. Estos conteos se llevaron a cabo durante una semana, en horarios pico establecidos de 6:30 a 8:30, de 12:00 a 14:00 y de 17:00 a 19:00.

\section{- Volumen motorizado}

Figura 17. Resultado primer periodo aforo motorizado con intervención

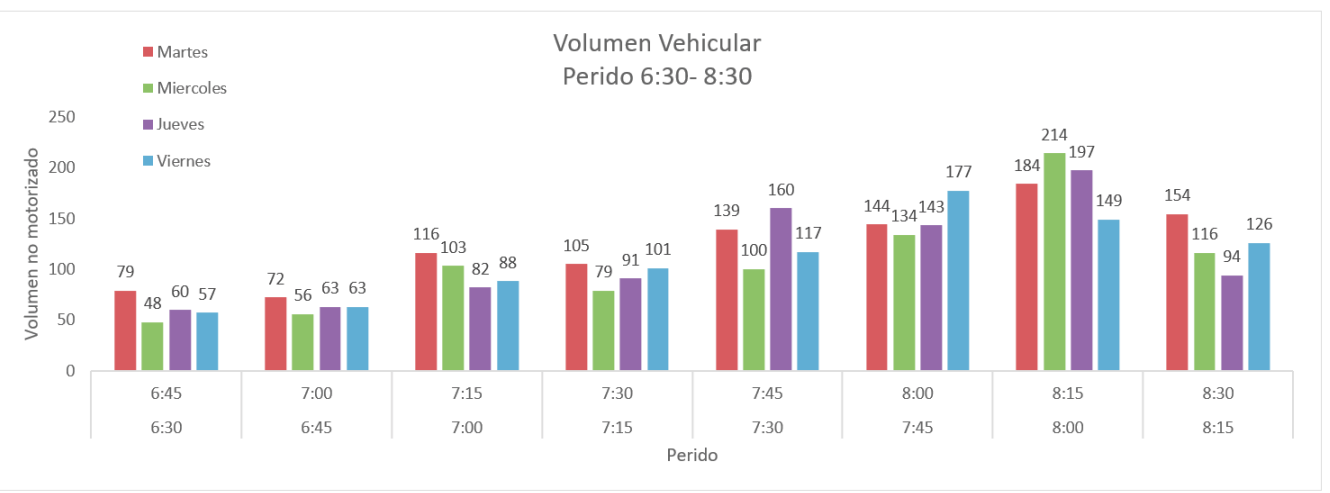

Fuente: autores

Figura 18. Resultado segundo periodo aforo motorizado con intervención

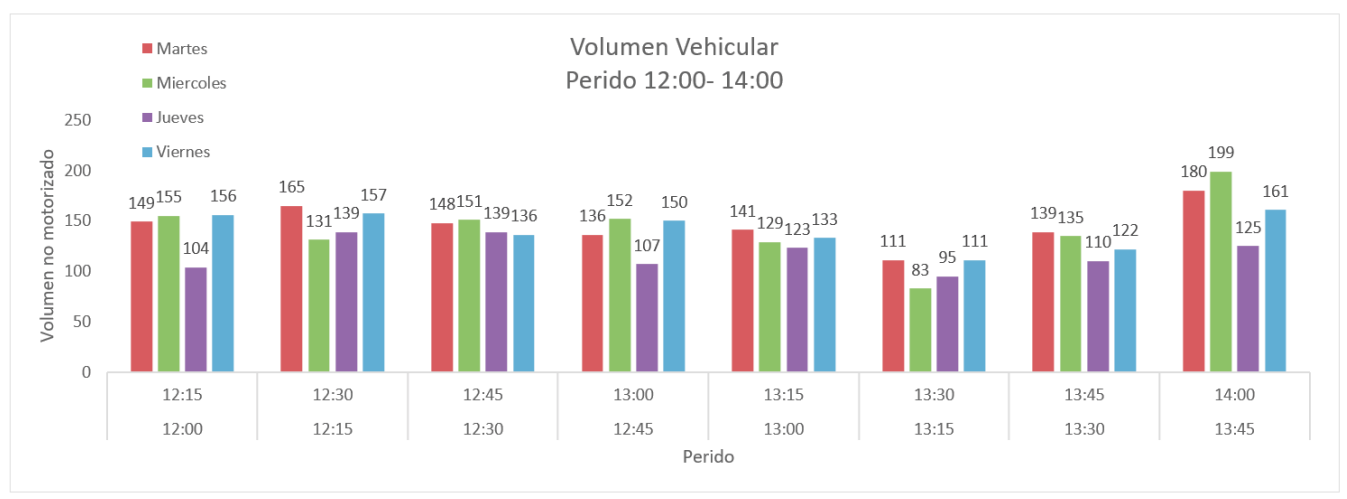

Fuente: autores 
Figura 19. Resultado tercer periodo aforo motorizado con intervención

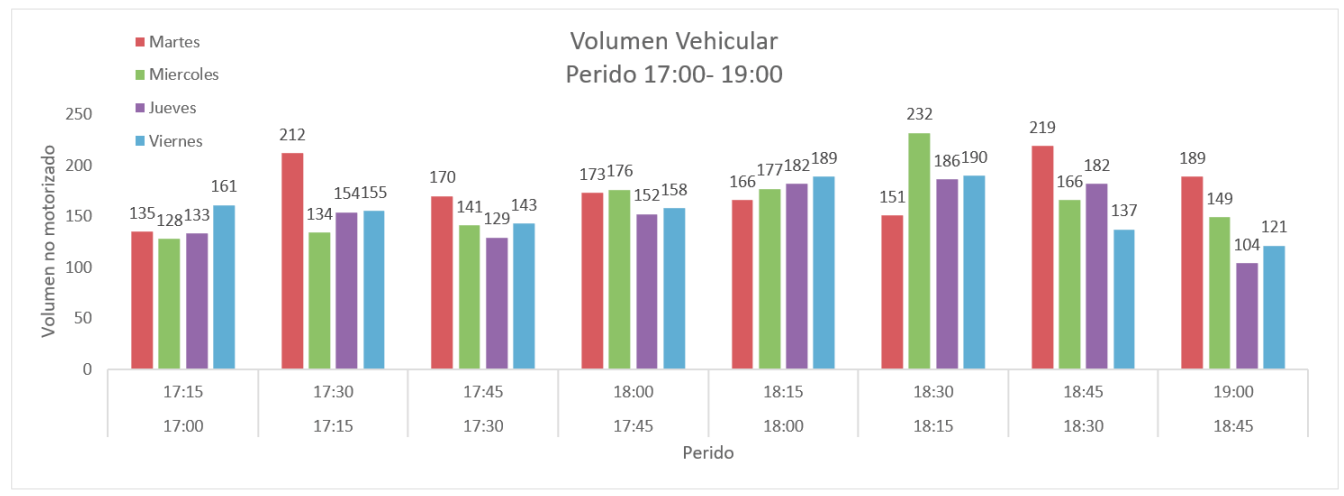

Fuente: autores

En el conteo de volumen motorizado se pudo apreciar lo siguiente: en la Figura 17, del periodo de 6:30 a 8:30, el flujo vehicular más alto se observa entre el horario de 8:00 a 8:15 del miércoles con una cantidad de 214 vehículos que transitan por la calle. El volumen vehicular del medio día se estudia en el periodo de 12:00 a 14:00 (ver Figura 18), en donde se observa que el mayor flujo vehicular se presenta en el horario de 13:45 a 14:00 en toda la semana con un total de 199 vehículos el miércoles, 180 el martes y 161 el lunes. El periodo con más flujo de vehículos motorizados es de 17:00 a 19:00, con un total de 232 vehículos el miércoles (ver Figura 19).

- Volumen motorizado

Figura 20. Resultado primer periodo aforo no motorizado con intervención

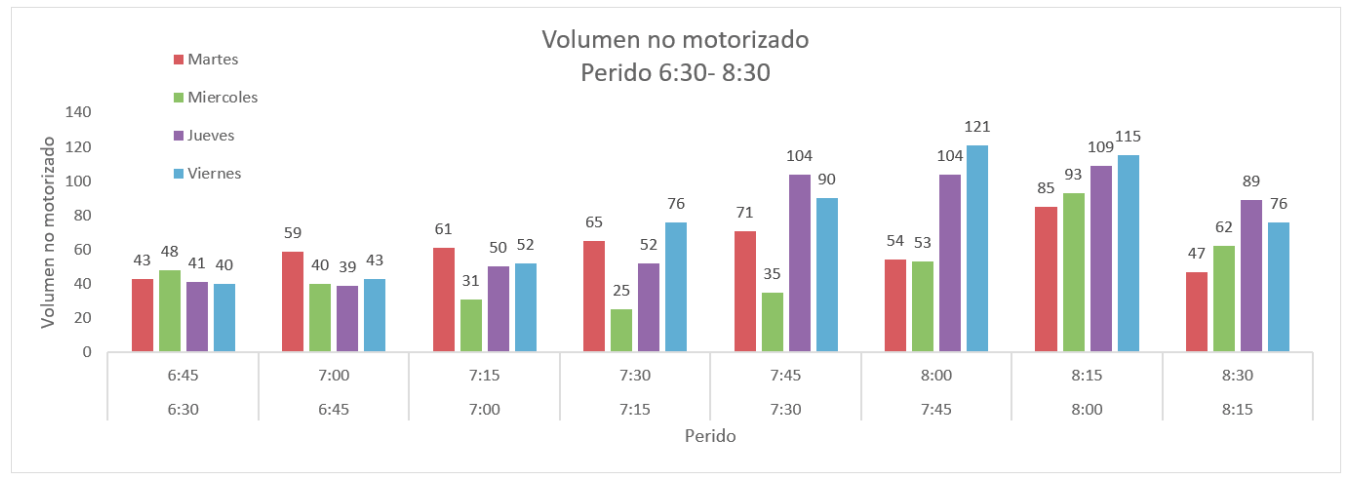

Fuente: autores 
INDAGA]E e-ISSN: 2357-5042 • Número 8 (2020) • Universidad de Ibagué • doi: https://doi.org/10.35707/indagare/809

Figura 21. Resultado segundo periodo aforo no motorizado con intervención

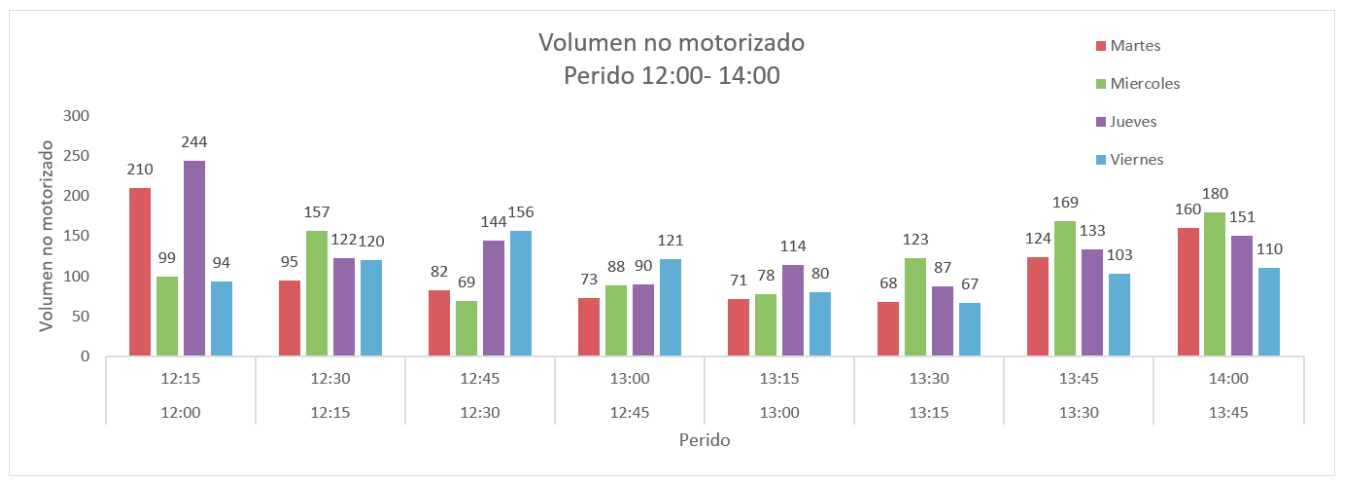

Fuente: autores

Figura 22. Resultado tercer periodo aforo no motorizado con intervención

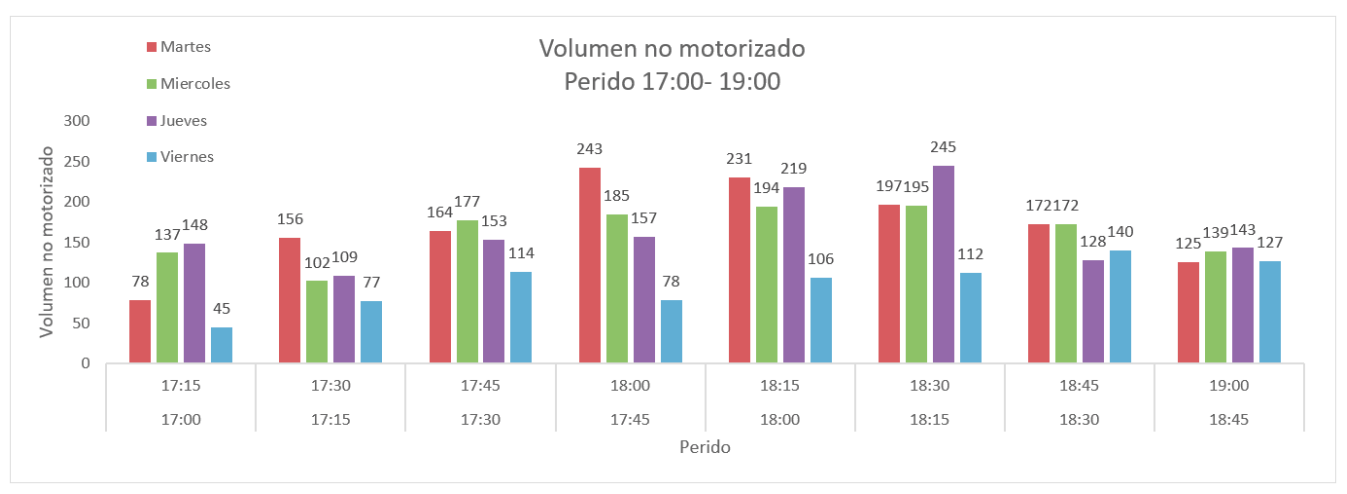

Fuente: autores

En este conteo se registraron los peatones y ciclistas que transitan por la calle. En la primera parte del día, en el periodo de 6:30 a 8:30, se presenta un total de 121 personas o biciusuarios para el miércoles en el horario de 7:45 a 8:00 (ver Figura 20). El día lunes, en el periodo de 12:00 a 14:00, se presenta un flujo no motorizado de 244 personas en el horario de 12:00 a 12:15 (ver Figura 21). El periodo con más flujo de no motorizados es 17:00 a 19:00, con un total de 245 personas o biciusuarios para el jueves (ver Figura 22). En ese mismo periodo se presenta un flujo de 186 motorizados (ver Figura 19). Es evidente el conflicto que se presenta en esa franja de horario, entre motorizados y no motorizados; esto se debe a que a esa hora muchos estudiantes y funcionarios terminan sus jornadas. 
- Ocupación visual taxi

Figura 23. Resultado ocupación visual taxi

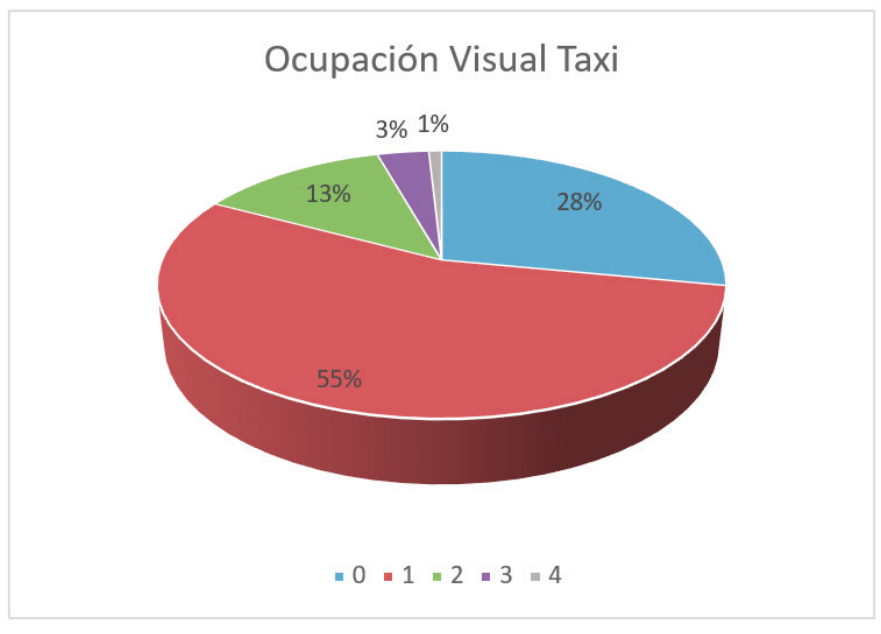

Fuente: autores

El $55 \%$ de los taxis que transitan por la calle 66 movilizan a solo un pasajero y el $28 \%$ no movilizan a ninguno, lo cual indica que una gran mayoría de estudiantes y funcionarios de la universidad lo emplean como su medio de transporte.

- Ocupación visual automóvil

Figura 24. Resultado ocupación visual automóvil

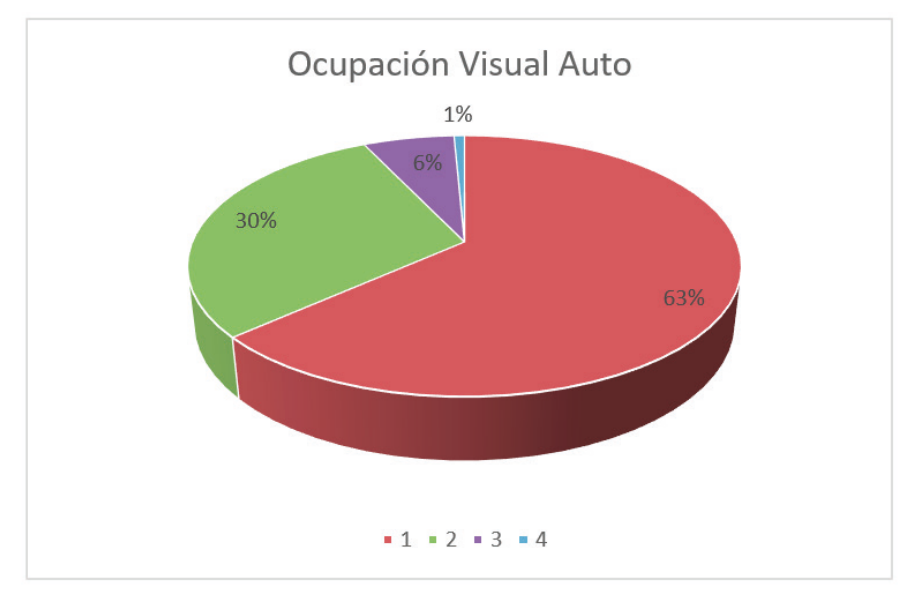

Fuente: autores 
Según los registros aforados en este formato, el $63 \%$ de los automóviles se movilizan con solo un pasajero; el conductor, y solo en el $30 \%$ de los automóviles se movilizan dos personas.

- Conflicto vehículos-peatones con intervención

Figura 25. Promedio vehicular y peatonal con intervención

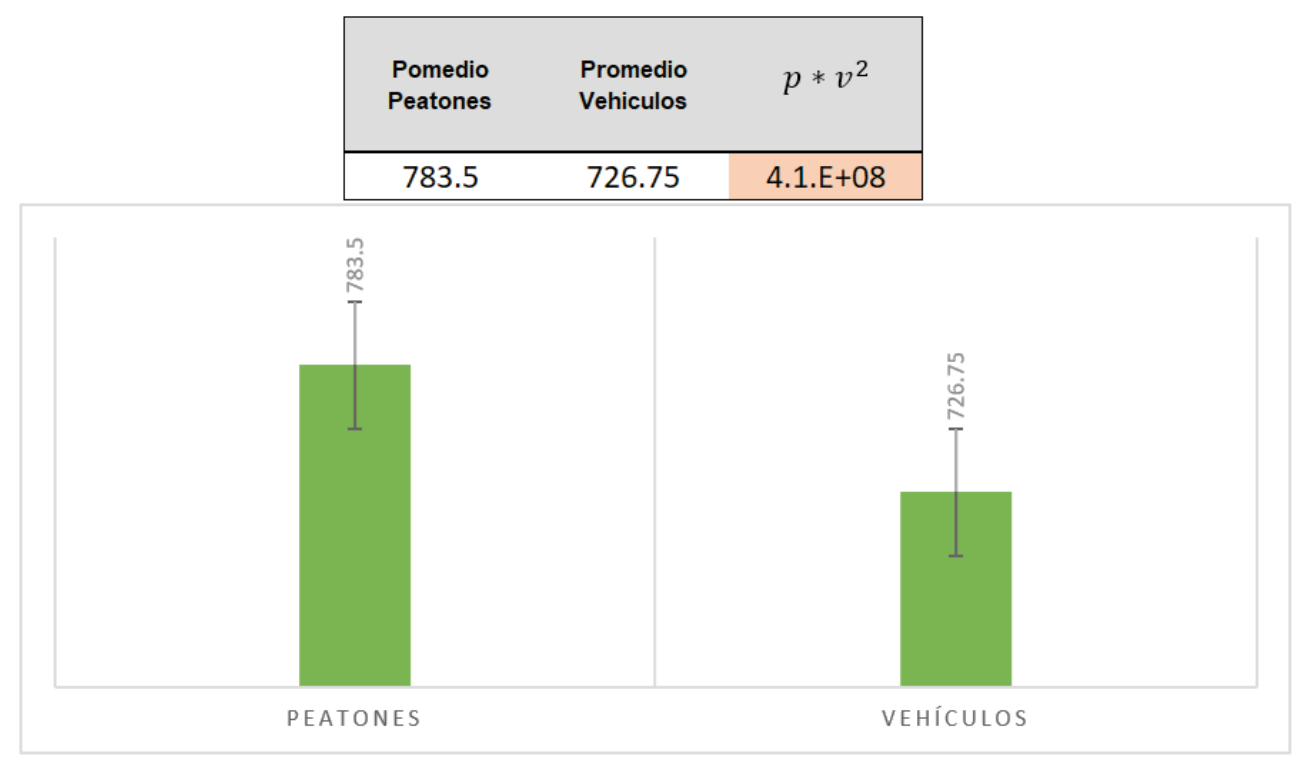

Fuente: autores

Respecto al conflicto vehículo-peatón, luego de cumplir con la intervención, el nivel de intensidad del conflicto se clasifica como severo, con promedio de 783.5 peatones y 726.75 vehículos.

\subsection{Intervención de urbanismo táctico}

El diseño final tuvo una transformación en el proceso constructivo por falta de recursos; pero la idea de la intervención se mantuvo y era permitir la movilidad de los diferentes actores, respetando las diversas formas de movilidad urbana en escala pequeña de calle. Se logró integrar los diferentes tipos de movilidad, además de mejorar la imagen urbana, debido a que se rescataron los espacios públicos de convivencia. El diseño tuvo un impacto porque permitió la interacción entre vehículos, ciclistas, peatones y transporte público, ofreciendo condiciones óptimas para cada uno de ellos. 
Figura 26. Infografía urbanismo táctico
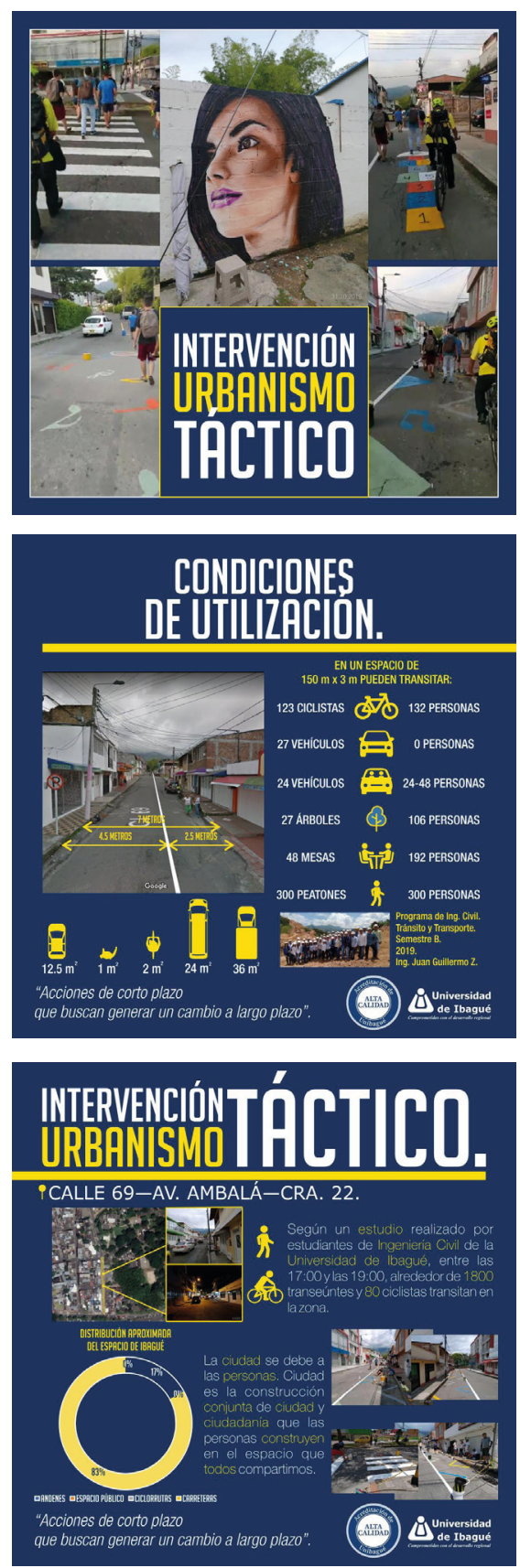

Fuente: Universidad de Ibagué (2019) 


\section{Potencial uso}

- Permitir a la comunidad llevar a cabo estas transformaciones necesarias dan cuenta del derecho que todos los ciudadanos tienen como participantes en la transformación de ciudad; en este sentido, es importante que cada persona sea consciente de la importancia de su opinión en los procesos que conllevan construir ciudad, pues son la fuente principal para el reconocimiento de la problemática, además de ser los principales beneficiados de estas intervenciones.

- En los aforos realizados luego de la intervención, no se tuvo en cuenta el formato de velocidades debido a que el resultado inicial demostró que la velocidad de diseño de la calle no era excedida de 30 kilómetros por hora, puesto que esta es la velocidad recomendada para zonas escolares y residenciales según el Ministerio de Transporte; sin embargo, se espera que con la implementación del diseño los conductores reduzcan la velocidad.

- El aforo del formato de ocupación visual se llevó a cabo luego de la intervención, debido a que se desconocía el número de pasajeros que se transportaban en un solo automóvil. Este es un valor de suma relevancia para conocer las condiciones de utilización de la calle. En consecuencia, se analiza que el espacio utilizado por los vehículos es mal empleado porque en los resultados se observa que en el $63 \%$ de los mismos se transporta una sola persona (un vehículo ocupa un espacio de $12.5 \mathrm{~m}^{\wedge} 2$ ) $y$, en ese espacio, doce personas podrían estar transitando como peatones. Lo anterior demuestra que el espacio de reducción de la vía para los automóviles es necesario, debido a que, en promedio, 800 peatones transitan por hora en la zona con solo un $17 \%$ de espacio para su movilidad.

- Este proyecto recoge los aprendizajes de los últimos semestres de estudio para exponer al público las posibilidades que hay a la hora de generar estrategias públicas que mitiguen problemas sociales, ambientales y urbanos. A la vez, logra plantear una forma metódica y ordenada de acciones que se pueden generalizar en la ciudad para su fácil aplicación y replicabilidad en distintos escenarios urbanos. De igual forma, permite entender la infraestructura vial no solo como un espacio, sino como la capacidad para entender la comunidad y sus necesidades.

- Este prototipo urbano de corto plazo contribuye a vislumbrar su efecto a largo plazo. Esta intervención permitió probar su eficacia de forma rápida y económica. Muchas de estas intervenciones han logrado institucionalizarse dentro de las políticas públicas municipales y departamentales, y replicarse en otros contextos donde sea necesario. 
INDAGA:ZE e-ISSN: 2357-5042 • Número 8 (2020) • Universidad de Ibagué • doi: https://doi.org/10.35707/indagare/809

- La solución a las múltiples problemáticas que presenta la movilidad se puede solventar en conjunto con la Subdirección de Seguridad Vial de la Secretaría de Movilidad y las administraciones municipales y departamentales, dado que en esta implementación no se pudo realizar el diseño propuesto por falta de recursos. Sin embargo, estas implementaciones deberían tener el apoyo de dichas entidades para lograr mejores resultados y emplear escalas mayores, enfocadas en un mismo objetivo que es la seguridad vial.

- Esta intervención no solo resuelve la problemática de movilidad, también impulsa el cuidado del medio ambiente, fomenta el desarrollo local y empodera a los habitantes en el desarrollo urbano.

- La sostenibilidad y mejoramiento de estas intervenciones urbanas dependen, en gran parte, de la activación humana. La universidad y la comunidad deben lograr un nivel de apropiación para que el uso, el cuidado y el mantenimiento de estos espacios urbanos esté a su cargo y no se limite solo a la intervención realizada.

\section{Ficha técnica del proyecto}

Título del proyecto: Aplicación de urbanismo táctico en la Universidad de Ibagué (Colombia).

PRIT: Desarrollo regional inclusivo y sustentable.

Código del proyecto: N/A.

Palabras claves: Peatón, urbanismo, movilidad, urbanismo táctico, intervención, diseño.

Grupo de investigación: GMAE.

Investigador principal: Juanita Ciro Torres.

Correo electrónico: juanitacirot@gmail.com

\section{Referencias}

Decreto 015 de 2011. (06 de enero de 2011). Diario Oficial, (47.944). Recuperado de https://n9.cl/rtxzy Gehl, J. (2010) Cities for people. Washington, D. C.: Island Press.

Gehl, J., \& Svarre, B. (2013). How to study public life. Washington, D. C.: Island Press.

Ministerio de Transporte. (2015). Manual de señalización vial. Dispositivos uniformes para la regulación del tránsito en calles, carreteras y ciclorrutas de Colombia. Bogota, D. C: Diseño Tremens.

National Association of City Transportation Officials. (2016). Global Street Design Guide. New York: Nacto. 